\title{
More on effective potentials for revolving D-branes
}

\author{
Satoshi Iso, ${ }^{a, b}$ Noriaki Kitazawa, ${ }^{c}$ Hikaru Ohta ${ }^{a}$ and Takao Suyama ${ }^{a}$ \\ ${ }^{a}$ Theory Center, High Energy Accelerator Research Organization (KEK), \\ Tsukuba, Ibaraki 305-0801, Japan \\ ${ }^{b}$ Graduate University for Advanced Studies (SOKENDAI), \\ Tsukuba, Ibaraki 305-0801, Japan \\ ${ }^{c}$ Department of Physics, Tokyo Metropolitan University, \\ Hachioji, Tokyo 192-0397, Japan \\ E-mail: iso@post.kek.jp, noriaki.kitazawa@tmu.ac.jp, \\ hohta@post.kek.jp, tsuyama@post.kek.jp
}

ABSTRACT: We continue to investigate the effective potential between a pair of $\mathrm{D} p$-branes revolving around each other by using the technique of partial modular transformation developed in our previous work. We determine the shape of the potential for general $p$ for a wide range of regions interpolating smaller and larger distances than the string scale $l_{s}$. We also discuss the backreaction of the D-brane system to the space-time metric and the validity of our calculations.

KEYwORDS: D-branes, p-branes

ARXIV EPRINT: 2006.01501 


\section{Contents}

1 Introduction 1

2 Partial modular transformation 2

3 Effective potential for small $\omega$

3.1 Contributions from SYM 4

3.2 Contributions from SUGRA 8

4 Shape of the effective potential $\quad 9$

$\begin{array}{lll}4.1 & \text { Multiple D } p \text {-branes } & 10\end{array}$

5 Conclusions and discussions $\quad 12$

$\begin{array}{lr}\text { A Various functions for SYM contributions } & 14\end{array}$

$\begin{array}{lll}\text { A.1 } f_{0, m}(x) & 15\end{array}$

$\begin{array}{ll}\text { A.2 } f_{1, m}(x) & 16\end{array}$

$\begin{array}{ll}\text { B Various functions for SUGRA contributions } & 17\end{array}$

$\begin{array}{lll}\text { B.1 } g_{3 / 2}(x) & 17\end{array}$

$\begin{array}{lll}\text { B.2 } & g_{2}(x) & 17\end{array}$

$\begin{array}{ll}\text { C } \mathcal{O}\left(\omega^{2}\right) \text { contributions for small } r & 18\end{array}$

\section{Introduction}

In this paper, we continue to investigate a system of D-branes which are revolving around each other discussed in [1]. Our motivation in these studies is to apply such a D-brane system to stringy phenomenological models of electroweak symmetry breaking [2-4], in which a small energy scale, compared to the UV stringy scale, may emerge due to a dynamical motion of D-branes. In particular, we hope to realize the weak scale as the radius $r$ of the circular orbit determined by the angular velocity of the revolving D-brane system $[5,6]$.

For this purpose, in [1] we developed an efficient method to calculate the effective potential between the revolving $\mathrm{D} p$-branes in a wide region of their distance, which we call partial modular transformation. By employing this method, we obtained an expression of the effective potential for general $p$ as a sum of complicated integrals, which will be recalled in section 3 . In [1], only the $p=3$ case was analyzed and the shape of the effective potential was explicitly obtained since this seems to be the most interesting from a phenomenological point of view. 
In this paper, we extend the analysis of the $p=3$ case to more general $\mathrm{D} p$-branes. Since the effective potential for large radius $r$ can be obtained rather easily by using the Type II supergravity (SUGRA), we focus on the region of $r$ smaller than the string scale $l_{s}:=\sqrt{2 \pi \alpha^{\prime}}$, where we need to use super Yang-Mills (SYM) theory as an effective theory. In particular, for $r \sim l_{s}$, we need to sum both contributions from supergravity and super Yang-Mills theory. If we simply sum these two, it causes a problem of double counting. The method of the partial modular transformation can avoid this problem, and we can obtain the effective potential in a good approximation interpolating from large $r>l_{s}$ to small $r<l_{s}$.

We find that the shape of the potential for general $\mathrm{D} p$-branes is qualitatively similar to the one for $p=3$, that is, an attractive force dominating the repulsive centrifugal force, appears for a certain situation. More specifically, the behaviors of the potentials for $p=0,1$ are different from the others. The result for $p=0$ indicates the presence of non-BPS bound states of D0-branes discussed in [7, 8]. We also discuss the validity of our calculation, in particular the effect of the backreaction of the whole D-brane system to the background space-time metric. The behavior of the potential at $r \ll l_{s}$ is also discussed.

The paper is organized as follows. In section 2, we recall the results of our previous paper [1]. The integral expressions of the effective potential are explicitly evaluated in section 3. The shape of the effective potential, including the centrifugal potential, is examined in section 4. The behavior of the effective potential for extremely small $r$ is analyzed in section 5. Some technical details are summarized in appendices.

\section{Partial modular transformation}

In this section, we briefly review our previous work [1]. First, we recall the method for calculating the effective potential between revolving D-branes.

Interactions between dynamical D-branes are mediated by exchange of closed strings. At the leading order in $g_{s}$, the effective potential can be also calculated by the one-loop amplitude of an open string stretched between the D-branes. Such calculations for static D-branes $[9,10]$ are well-known. Schematically, it is given as

$$
V(R)=\int_{0}^{\infty} \frac{d t}{t} Z_{\mathrm{open}}(t, R)
$$

where $R$ is the distance between the D-branes, which may vary with time in general. For D-branes in motion, calculations are more difficult to perform; when they move at a constant relative velocity, weak attractive force is obtained [11, 12]. For general motions, only perturbative calculations are performed $[13,14]$. When D-branes are revolving around each other, we obtained attractive force by using a perturbative method [15].

For performing the calculation in a more efficient way, we developed a new technique in [1]. We first rewrite this into the following sum of open and closed string contributions (see also [16]);

$$
V(R)=\int_{1}^{\infty} \frac{d t}{t} Z_{\text {open }}(t, R)+\int_{1}^{\infty} d s Z_{\text {closed }}(s, R)
$$


which is obtained from (2.1) by dividing the integration region into $[0,1)$ and $[1, \infty)$, and performing the modular transformation $s=t^{-1}$ only for the integration region over $[0,1)$. We call this operation the partial modular transformation. Note that $Z_{\text {closed }}(s, R)$ gives the amplitude for exchanging one closed string between the D-branes.

In this expression, we can safely ignore contributions from all heavy states in $Z_{\text {open }}(t, R)$ and $Z_{\text {closed }}(s, R)$ as far as a mass gap exits between lightest (massless) states and heavy massive states. It does hold irrespective of the value of $R$ from $R \gg l_{s}$ to $R \ll l_{s}$. Calculating the contributions of light states in both open and closed string sectors turns out to give an accurate approximation $\tilde{V}(R)$ for the effective potential $V(R)$. In [1], we see that its accuracy to the original potential $V(R)$ is just a few percents for an arbitrary value of $R$.

By construction, $\tilde{V}(R)$ is schematically given by the form

$$
\tilde{V}(R)=(1 \text {-loop in SYM })+(\text { tree-level in SUGRA })
$$

where each string contribution is replaced by its lowest modes. An important point here is that the Schwinger parameters $t, s$ have cut-offs which allow us to avoid a double-counting of contributions to $\tilde{V}(R)$. Note that the calculation of the right-hand side is possible even when we do not know how to quantize an open string stretched between the dynamical D-branes. It is also interesting to notice that the SUGRA contribution can be interpreted as threshold corrections to the SYM contribution since the former represents effects coming from heavy open string states.

In [1], we applied the above method to a pair of $\mathrm{D} p$-branes revolving around each other. Let $r$ and $\omega$ be the radius of the circular orbit and the angular velocity of the $\mathrm{D} p$-branes, respectively, so that we have $R=2 r$ in this case. We obtained an integral formula which gives a good approximation $\tilde{V}_{p}(2 r)$ for the effective potential between the revolving D $p$-branes. For a systematic evaluation of the potential, we divide $\tilde{V}_{p}(2 r)$ into the following three contributions: bosonic fields in open strings $\tilde{V}_{B, p}(2 r)$, fermionic fields in open strings $\tilde{V}_{F, p}(2 r)$ and closed string contributions $\tilde{V}_{c, p}(2 r)$,

$$
\tilde{V}_{p}(2 r)=\tilde{V}_{B, p}(2 r)+\tilde{V}_{F, p}(2 r)+\tilde{V}_{c, p}(2 r)
$$

The bosonic fields, including ghosts, in SYM give the contributions,

$$
\begin{aligned}
\tilde{V}_{B, p}(2 r)= & -\int_{\Lambda^{-2}}^{\infty} \frac{d t}{t} \int \frac{d^{p+1} k}{(2 \pi)^{p+1}} e^{-t\left(k^{2}+4 r^{2}\right)} \\
& \times\left[6+2 e^{-t\left(\omega^{2}-\frac{8(r \omega)^{2}}{k^{2}+4 r^{2}}\right)} \cosh \left(t \sqrt{4 \omega^{2} k_{\tau}^{2}+\left(\frac{8(r \omega)^{2}}{k^{2}+4 r^{2}}\right)^{2}}\right)\right],
\end{aligned}
$$

where $\Lambda:=1 / \sqrt{2 \pi \alpha^{\prime}}=1 / l_{s}$ and $k_{\tau}$ is the time component of Euclidean $(p+1)$-vector $k$. The fermionic fields in SYM give

$$
\tilde{V}_{F, p}(2 r)=8 \int_{\Lambda^{-2}}^{\infty} \frac{d t}{t} \int \frac{d^{p+1} k}{(2 \pi)^{p+1}} e^{-t\left(k^{2}+4 r^{2}\right)} e^{-\frac{1}{4} t \omega^{2}} \cosh \left(t \sqrt{\omega^{2} k_{\tau}^{2}+4(r \omega)^{2}}\right)
$$


and the contributions from the bosonic fields in Type II SUGRA are given by

$$
\begin{aligned}
\tilde{V}_{c, p}(2 r)= & -\kappa_{10}^{2} T_{p}^{2}(4 \pi)^{-\frac{10-p}{2}} \frac{(r \omega)^{2}}{1+(r \omega)^{2}} \int_{\tilde{\Lambda}^{-2}}^{\infty} d s s^{-\frac{10-p}{2}} \\
& \times \int_{-\infty}^{+\infty} d \zeta \exp \left[-\frac{1}{4 s}\left(\zeta^{2}+2 r^{2}(1+\cos \omega \zeta)\right)\right](1+\cos \omega \zeta)^{2},
\end{aligned}
$$

where $\tilde{\Lambda}:=2 / \sqrt{2 \pi \alpha^{\prime}}$, and $\kappa_{10}^{2} T_{p}^{2}=\pi\left(2 \pi l_{s}^{2}\right)^{3-p}$. These integrals have various subtle behaviors when we expand them as a power series of either $r / \omega$ or $\omega / r$. In the following, we explicitly evaluate them to analyze the shape of $\tilde{V}_{p}(2 r)$ for general $p$. The case $p=3$ was discussed in [1].

Note that the above expressions are derived in the Euclidean signature. When we discuss the dynamics of $\mathrm{D} p$-branes in this potential, we will perform a suitable analytic continuation of $\omega$. Note also that $r$ in $\tilde{V}_{B, p}(2 r)$ and $\tilde{V}_{F, p}(2 r)$ has the mass dimension +1 , while $r$ in $\tilde{V}_{c, p}(2 r)$ has -1 . Therefore, we will replace $r$ with $r / l_{s}^{2}$ for the former at the end of calculations. We believe that it will not make any confusion.

\section{Effective potential for small $\omega$}

In this section, we will evaluate the integrals in eqs. (2.5), (2.6) and (2.7) to investigate the shape of the approximate potential $\tilde{V}(2 r)$ for each $p$. In particular, we are interested in the situation where $\omega$ is small and the motion is nonrelativistic $r \omega \ll 1$. In the following, we expand the integrands in eqs. (2.5), (2.6) and (2.7) with respect to $\omega$, and then perform the integrals term by term.

\subsection{Contributions from SYM}

By rescaling the integration variables, the integrals eqs. (2.5) and (2.6) are functions of $\omega / r$, up to some overall factors, and we can expand them in terms of $\omega / r$. Therefore, the following calculations are valid as long as $\omega \ll r$ is satisfied.

First, let us consider $\tilde{V}_{B, p}(2 r)$. By expanding the integrands with respect to $\omega / r$, the $t$-integration can be performed and we have

$$
\begin{aligned}
\tilde{V}_{B, p}(2 r)= & c(r)+\int \frac{d^{p+1} k}{(2 \pi)^{p+1}} e^{-\left(k^{2}+4 r^{2}\right) / \Lambda^{2}}\left[\omega^{2}\left(\frac{2 \Lambda^{2}-4 k_{\tau}^{2}}{\Lambda^{2}\left(k^{2}+4 r^{2}\right)}-\frac{16 r^{2}+4 k_{\tau}^{2}}{\left(k^{2}+4 r^{2}\right)^{2}}\right)\right. \\
& +\omega^{4}\left(-\frac{3 \Lambda^{4}-12 \Lambda^{2} k_{\tau}^{2}+4 k_{\tau}^{4}}{3 \Lambda^{6}\left(k^{2}+4 r^{2}\right)}+\frac{16 \Lambda^{2} r^{2}-32 k_{\tau}^{2} r^{2}-\Lambda^{4}+8 k_{\tau}^{2} \Lambda^{2}-4 k_{\tau}^{4}}{\Lambda^{4}\left(k^{2}+4 r^{2}\right)^{2}}\right. \\
& \left.\left.-\frac{128 r^{4}-16 \Lambda^{2} r^{2}+64 k_{\tau}^{2} r^{2}-8 k_{\tau}^{2} \Lambda^{2}+8 k_{\tau}^{4}}{\Lambda^{2}\left(k^{2}+4 r^{2}\right)^{3}}-\frac{128 r^{4}+64 k_{\tau}^{2} r^{2}+8 k_{\tau}^{4}}{\left(k^{2}+4 r^{2}\right)^{4}}\right)\right] \\
& +\mathcal{O}\left(\omega^{6}\right),
\end{aligned}
$$


where $c(r)$ is an $\mathcal{O}\left(\omega^{0}\right)$ contribution. This term is canceled by an $\mathcal{O}\left(\omega^{0}\right)$ term in $\tilde{V}_{F, p}(2 r)$ since the system with $\omega=0$ is a BPS configuration. In the same manner, we obtain

$$
\begin{aligned}
\tilde{V}_{F, p}(2 r)= & -c(r)+\int \frac{d^{p+1} k}{(2 \pi)^{p+1}} e^{-\left(k^{2}+4 r^{2}\right) / \Lambda^{2}}\left[\omega^{2}\left(\frac{16 r^{2}-2 \Lambda^{2}+4 k_{\tau}^{2}}{\Lambda^{2}\left(k^{2}+4 r^{2}\right)}+\frac{16 r^{2}+4 k_{\tau}^{2}}{\left(k^{2}+4 r^{2}\right)^{2}}\right)\right. \\
& +\omega^{4}\left(\frac{64 r^{4}-48 \Lambda^{2} r^{2}+32 k_{\tau}^{2} r^{2}+3 \Lambda^{4}-12 k_{\tau}^{2} \Lambda^{2}+4 k_{\tau}^{4}}{12 \Lambda^{6}\left(k^{2}+4 r^{2}\right)}\right. \\
& +\frac{64 r^{4}-32 \Lambda^{2} r^{2}+32 k_{\tau}^{2} r^{2}+\Lambda^{4}-8 k_{\tau}^{2} \Lambda^{2}+4 k_{\tau}^{4}}{4 \Lambda^{4}\left(k^{2}+4 r^{2}\right)^{2}} \\
& \left.\left.+\frac{32 r^{4}-8 \Lambda^{2} r^{2}+16 k_{\tau}^{2} r^{2}-2 k_{\tau}^{2} \Lambda^{2}+2 k_{\tau}^{4}}{\Lambda^{2}\left(k^{2}+4 r^{2}\right)^{3}}+\frac{32 r^{4}+16 k_{\tau}^{2} r^{2}+2 k_{\tau}^{4}}{\left(k^{2}+4 r^{2}\right)^{4}}\right)\right] \\
& +\mathcal{O}\left(\omega^{6}\right) .
\end{aligned}
$$

Summing them, we obtain the following SYM part of the potential,

$$
\begin{aligned}
\tilde{V}_{o, p}(2 r):= & \tilde{V}_{B, p}(2 r)+\tilde{V}_{F, p}(2 r) \\
= & \int \frac{d^{p+1} k}{(2 \pi)^{p+1}} e^{-\left(k^{2}+4 r^{2}\right) / \Lambda^{2}}\left[\omega^{2} \frac{16 r^{2}}{\Lambda^{2}\left(k^{2}+4 r^{2}\right)}\right. \\
& +\omega^{4}\left(\frac{-9 \Lambda^{4}-48 r^{2} \Lambda^{2}+64 r^{4}+\left(36 \Lambda^{2}+32 r^{2}\right) k_{\tau}^{2}-12 k_{\tau}^{4}}{12 \Lambda^{6}\left(k^{2}+4 r^{2}\right)}\right. \\
& +\frac{-3 \Lambda^{4}+32 \Lambda^{2} r^{2}+64 r^{4}+\left(24 \Lambda^{2}-96 r^{2}\right) k_{\tau}^{2}-12 k_{\tau}^{4}}{4 \Lambda^{4}\left(k^{2}+4 r^{2}\right)^{2}} \\
& \left.\left.+\frac{8 \Lambda^{2} r^{2}-96 r^{4}+\left(6 \Lambda^{2}-48 r^{2}\right) k_{\tau}^{2}-6 k_{\tau}^{4}}{\Lambda^{2}\left(k^{2}+4 r^{2}\right)^{3}}+\frac{-96 r^{4}-48 k_{\tau}^{2} r^{2}-6 k_{\tau}^{4}}{\left(k^{2}+4 r^{2}\right)^{4}}\right)\right] \\
& +\mathcal{O}\left(\omega^{6}\right) .
\end{aligned}
$$

To perform the $k$-integration, it is convenient to use the following formula,

$$
\int \frac{d^{p+1} k}{(2 \pi)^{p+1}} f\left(k^{2}\right) k_{\tau}^{2 m}=\frac{1}{\pi}(4 \pi)^{-\frac{p}{2}} \frac{\Gamma\left(m+\frac{1}{2}\right)}{\Gamma\left(m+\frac{p+1}{2}\right)} \int_{0}^{\infty} d k f\left(k^{2}\right) k^{2 m+p},
$$

where $k_{\tau}$ is a Euclidean-time component of the momentum $k$. This is obtained by employing the polar coordinates for $k$ and performing the integration for the angular coordinates. Then, we obtain

$$
\begin{aligned}
\tilde{V}_{o, p}(2 r)= & \frac{1}{\pi}(4 \pi)^{-\frac{p}{2}} \frac{\sqrt{\pi}}{\Gamma\left(\frac{p+1}{2}\right)} \int_{0}^{\infty} d k e^{-\left(k^{2}+4 r^{2}\right) / \Lambda^{2}} \\
& \times\left[\omega^{2} \frac{16 r^{2}}{\Lambda^{2}} \frac{k^{p}}{k^{2}+4 r^{2}}+\omega^{4} \sum_{m=-1}^{4} \frac{c_{m}(2 r / \Lambda)}{\Lambda^{4-2 m}} \frac{k^{p}}{\left(k^{2}+4 r^{2}\right)^{m}}\right]+\mathcal{O}\left(\omega^{6}\right),
\end{aligned}
$$


where the functions $c_{m}(x)$ are defined as

$$
\begin{aligned}
c_{-1}(x) & :=-g_{4}, \\
c_{0}(x) & :=\frac{6 g_{4}+2 g_{2}}{3} x^{2}+3\left(g_{2}-g_{4}\right), \\
c_{1}(x) & :=-\frac{3 g_{4}+2 g_{2}-1}{3} x^{4}+\left(6 g_{4}-9 g_{2}-1\right) x^{2}-\frac{24 g_{4}-24 g_{2}+3}{4}, \\
c_{2}(x) & :=\left(-3 g_{4}+6 g_{2}+1\right) x^{4}+\left(12 g_{4}-18 g_{2}+2\right) x^{2}-\frac{24 g_{4}-24 g_{2}+3}{4}, \\
c_{3}(x) & :=-\left(6 g_{4}-12 g_{2}+6\right) x^{4}+\left(12 g_{4}-18 g_{2}+2\right) x^{2}, \\
c_{4}(x) & :=-\left(6 g_{4}-12 g_{2}+6\right) x^{4},
\end{aligned}
$$

and $g_{2}:=1 /(p+1), g_{4}:=3 /(p+1)(p+3)$. By defining the following functions

$$
f_{p, m}(x):=x^{1+p-2 m} \int_{0}^{\infty} d k e^{-x^{2}\left(k^{2}+1\right)} \frac{k^{p}}{\left(k^{2}+1\right)^{m}},
$$

$\tilde{V}_{o, p}(2 r)$ can be rewritten as

$$
\begin{aligned}
\tilde{V}_{o, p}(2 r)= & \frac{1}{\pi}(4 \pi)^{-\frac{p}{2}} \frac{\sqrt{\pi}}{\Gamma\left(\frac{p+1}{2}\right)}\left[4 \omega^{2} \Lambda^{p-1}\left(\frac{2 r}{\Lambda}\right)^{2} f_{p, 1}\left(\frac{2 r}{\Lambda}\right)+\omega^{4} \Lambda^{p-3} \sum_{m=-1}^{4} c_{m}\left(\frac{2 r}{\Lambda}\right) f_{p, m}\left(\frac{2 r}{\Lambda}\right)\right] \\
& +\mathcal{O}\left(\omega^{6}\right) .
\end{aligned}
$$

Note that the functions $f_{p, m}(x)$ satisfy the following recurrence relation,

$$
f_{p, m}(x)=f_{p-2, m-1}(x)-x^{2} f_{p-2, m}(x) .
$$

Thus, it is enough to determine $f_{0, m}(x)$ or $f_{1, m}(x)$ depending on whether $p$ is even or odd. The details of these functions are given in appendix A.

We apply the formulas given in appendix A, and replace $r$ by $r / l_{s}^{2}$ such that $r$ has the dimension of length. Returning to the Lorentzian signature by performing the analytic continuation of $\omega$, the potential is obtained as follows. For even $p$,

$$
\begin{aligned}
& \tilde{V}_{o, 0}(2 r)=-\frac{1}{\sqrt{\pi}}\left[4 \sqrt{\pi} \omega^{2} r\left(1-\operatorname{Erf}\left(\frac{2 r}{l_{s}}\right)\right)\right. \\
&\left.-\omega^{4}\left\{\left(-\frac{15 l_{s}^{5}}{32 r^{2}}-\frac{5 l_{s}^{3}}{4}+\frac{34 l_{s} r^{2}}{3}\right) e^{-4 r^{2} / l_{s}^{2}}-\sqrt{\pi}\left(\frac{15 l_{s}^{6}}{128 r^{3}}+\frac{64 r^{3}}{3}\right)\left(1-\operatorname{Erf}\left(\frac{2 r}{l_{s}}\right)\right)\right\}\right] \\
&+ \mathcal{O}\left(\omega^{6}\right), \\
& \tilde{V}_{o, 2}(2 r)=-\frac{1}{\pi^{3 / 2}}\left[\omega^{2}\left\{\frac{4 r^{2}}{l_{s}^{3}} e^{-4 r^{2} / l_{s}^{2}}-\frac{8 \sqrt{\pi} r^{3}}{l_{s}^{4}}\left(1-\operatorname{Erf}\left(\frac{2 r}{l_{s}}\right)\right)\right\}\right. \\
&-\omega^{4}\left\{\left(-\frac{3 l_{s}}{16}-\frac{37 r^{2}}{18 l_{s}}-\frac{128 r^{4}}{9 l_{s}^{3}}\right) e^{-4 r^{2} / l_{s}^{2}}\right. \\
&\left.\left.+\sqrt{\pi}\left(-\frac{3 l_{s}^{2}}{64 r}+\frac{8 r^{3}}{l_{s}^{2}}+\frac{256 r^{5}}{9 l_{s}^{4}}\right)\left(1-\operatorname{Erf}\left(\frac{2 r}{l_{s}}\right)\right)\right\}\right]+\mathcal{O}\left(\omega^{6}\right),
\end{aligned}
$$




$$
\begin{gathered}
\tilde{V}_{o, 4}(2 r)=-\frac{1}{\pi^{5 / 2}}\left[\omega^{2} \frac{r^{2}}{l_{s}^{5}}\left\{\frac{1}{3}\left(1-\frac{8 r^{2}}{l_{s}^{2}}\right) e^{-4 r^{2} / l_{s}^{2}}+\frac{16 \sqrt{\pi} r^{3}}{3 l_{s}^{3}}\left(1-\operatorname{Erf}\left(\frac{2 r}{l_{s}}\right)\right)\right\}\right. \\
-\omega^{4} \frac{r}{l_{s}^{2}}\left\{\left(-\frac{9 r}{40 l_{s}}+\frac{64 r^{3}}{15 l_{s}^{3}}+\frac{128 r^{5}}{15 l_{s}^{5}}\right) e^{-4 r^{2} / l_{s}^{2}}\right. \\
\left.\left.-\sqrt{\pi}\left(\frac{1}{32}+\frac{32 r^{4}}{3 l_{s}^{4}}+\frac{256 r^{6}}{15 l_{s}^{6}}\right)\left(1-\operatorname{Erf}\left(\frac{2 r}{l_{s}}\right)\right)\right\}\right]+\mathcal{O}\left(\omega^{6}\right), \\
\tilde{V}_{o, 6}(2 r)=-\frac{1}{\pi^{7 / 2}}\left[\omega^{2} \frac{r^{2}}{l_{s}^{7}}\left\{\left(\frac{1}{20}-\frac{2 r^{2}}{15 l_{s}^{2}}+\frac{16 r^{4}}{15 l_{s}^{4}}\right) e^{-4 r^{2} / l_{s}^{2}}-\frac{32 \sqrt{\pi} r^{5}}{15 l_{s}^{5}}\left(1-\operatorname{Erf}\left(\frac{2 r}{l_{s}}\right)\right)\right\}\right. \\
-\frac{\omega^{4}}{l_{s}^{3}}\left\{\left(-\frac{11 r^{2}}{840 l_{s}^{2}}+\frac{26 r^{4}}{105 l_{s}^{4}}-\frac{176 r^{6}}{63 l_{s}^{6}}-\frac{1024 r^{8}}{315 l_{s}^{8}}\right) e^{-4 r^{2} / l_{s}^{2}}\right. \\
\left.\left.+\frac{\sqrt{\pi} r^{3}}{l_{s}^{3}}\left(-\frac{1}{16}+\frac{32 r^{4}}{5 l_{s}^{4}}+\frac{2048 r^{6}}{315 l_{s}^{6}}\right)\left(1-\operatorname{Erf}\left(\frac{2 r}{l_{s}}\right)\right)\right\}\right] \\
+\mathcal{O}\left(\omega^{6}\right), \quad
\end{gathered}
$$

and for odd $p$,

$$
\begin{aligned}
\tilde{V}_{o, 1}(2 r)= & -\frac{1}{\pi}\left[\omega^{2} \frac{4 r^{2}}{l_{s}^{2}} E_{1}\left(4 r^{2} / l_{s}^{2}\right)\right. \\
& \left.-\omega^{4}\left\{\left(-\frac{l_{s}^{4}}{8 r^{2}}-\frac{l_{s}^{2}}{2}+\frac{13 r^{2}}{3}\right) e^{-4 r^{2} / l_{s}^{2}}+\left(-2 r^{2}-\frac{16 r^{4}}{l_{s}^{2}}\right) E_{1}\left(4 r^{2} / l_{s}^{2}\right)\right\}\right] \\
& +\mathcal{O}\left(\omega^{6}\right), \\
\tilde{V}_{o, 3}(2 r)= & -\frac{1}{\pi^{2}}\left[\omega^{2}\left\{\frac{r^{2}}{l_{s}^{4}} e^{-4 r^{2} / l_{s}^{2}}-\frac{4 r^{4}}{l_{s}^{6}} E_{1}\left(4 r^{2} / l_{s}^{2}\right)\right\}\right. \\
& \left.-\omega^{4}\left\{\left(-\frac{1}{16}-\frac{7 r^{2}}{12 l_{s}^{2}}-\frac{10 r^{4}}{3 l_{s}^{4}}\right) e^{-4 r^{2} / l_{s}^{2}}+\left(\frac{6 r^{4}}{l_{s}^{4}}+\frac{40 r^{6}}{3 l_{s}^{6}}\right) E_{1}\left(4 r^{2} / l_{s}^{2}\right)\right\}\right] \\
& +\mathcal{O}\left(\omega^{6}\right), \\
\tilde{V}_{o, 5}(2 r)= & -\frac{1}{\pi^{3}}\left[\omega^{2}\left\{\left(\frac{r^{2}}{8 l_{s}^{6}}-\frac{r^{4}}{2 l_{s}^{8}}\right) e^{-4 r^{2} / l_{s}^{2}}+\frac{2 r^{6}}{l_{s}^{10}} E_{1}\left(4 r^{2} / l_{s}^{2}\right)\right\}\right. \\
& \left.-\omega^{4}\left\{\left(-\frac{7 r^{2}}{72 l_{s}^{4}}+\frac{31 r^{4}}{36 l_{s}^{6}}+\frac{14 r^{6}}{9 l_{s}^{8}}\right) e^{-4 r^{2} / l_{s}^{2}}+\left(-\frac{5 r^{6}}{l_{s}^{8}}-\frac{56 r^{8}}{9 l_{s}^{10}}\right) E_{1}\left(4 r^{2} / l_{s}^{2}\right)\right\}\right] \\
& +\mathcal{O}\left(\omega^{6}\right), \\
& +\frac{1}{\pi^{4}}\left[\omega^{2}\left\{\left(\frac{r^{2}}{48 l_{s}^{8}}-\frac{r^{4}}{24 l_{s}^{10}}+\frac{r^{6}}{6 l_{s}^{12}}\right) e^{-4 r^{2} / l_{s}^{2}}-\frac{2 r^{8}}{3 l_{s}^{14}} E_{1}\left(4 r^{2} / l_{s}^{2}\right)\right\}\right. \\
& -\omega^{4}\left\{\left(-\frac{r^{2}}{192 l_{s}^{6}}+\frac{r^{4}}{12 l_{s}^{8}}-\frac{11 r^{6}}{24 l_{s}^{10}}-\frac{r^{8}}{2 l_{s}^{12}}\right) e^{-4 r^{2} / l_{s}^{2}}\right. \\
& \left.\left.\left.+\frac{7 r^{8}}{3 l_{s}^{12}}+\frac{2 r^{10}}{l_{s}^{14}}\right) E_{1}\left(4 r^{2} / l_{s}^{2}\right)\right\}\right]+\mathcal{O}\left(\omega^{6}\right) . \\
\tilde{V}_{o, 7}(2 r) & (3.21) \\
&
\end{aligned}
$$




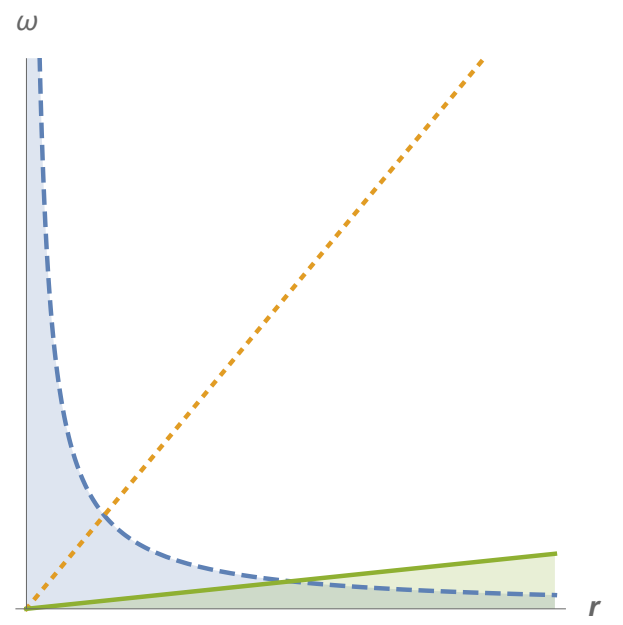

Figure 1. Validity regions of SUGRA and SYM calculations. In blue region, the nonrelativistic condition, $\omega \ll 1 / r$, is satisfied and our SUGRA calculation is valid. On the other hand, in the green region, the SYM calculations in terms of $\omega l_{s}^{2} / r$ expansion is valid. We also depicted a line of $\omega=2 r / \sqrt{3}$, beyond which an instability may occur (see discussions in section 5 ). Thus, our analysis is valid in the overlapping region near the $\omega=0$ line.

\subsection{Contributions from SUGRA}

In the following, we expand the integrand of the formula (2.7) with respect to $\omega$ in order to simplify the expression. It is obvious from the expression that the expansion starts with $\mathcal{O}\left(\omega^{4}\right)$ terms. Therefore, the contributions from SUGRA fields are sub-leading when $\omega$ is small.

By a suitable rescaling of the integration variable, we find that the integrand depends only on the combination $v=r \omega$, up to some overall factor. Therefore, the following calculations are valid in a nonrelativistic region where $\omega \ll 1 / r$ is satisfied. In figure 1 , we draw this validity region as well as the validity region of the SYM calculations $\omega \ll r / l_{s}^{2}$ discussed in section 3.1. There are overlaps of these two regions, and hence our analysis is valid there.

The leading order of the expansion is given by

$$
\tilde{V}_{c, p}(2 r)=-\kappa_{10}^{2} T_{p}^{2}(4 \pi)^{-(9-p) / 2} \tilde{\Lambda}^{7-p}(r \omega)^{4} g_{(9-p) / 2}\left(\frac{r \tilde{\Lambda}}{\sqrt{2}}\right)+\mathcal{O}\left(\omega^{6}\right),
$$

where

$$
g_{\alpha}(x):=\int_{1}^{\infty} d s s^{-\alpha} e^{-x^{2} / s},
$$

and we have performed the analytic continuation of $\omega$, which is in fact trivial. Note that the integral for $g_{\alpha}(x)$ is well-defined for $\alpha>1$, that is, $p<7$. They satisfy the following recurrence relations

$$
g_{\alpha}(x)=\frac{\alpha-2}{x^{2}} g_{\alpha-1}(x)-\frac{1}{x^{2}} e^{-x^{2}} .
$$

Therefore, it is enough to determine $g_{3 / 2}(x)$ or $g_{2}(x)$ depending on whether $p$ is even or odd. The details of these functions are given in appendix B. According to this, the potential for 
even $p$ is obtained as

$$
\begin{aligned}
\tilde{V}_{c, 0}(2 r)= & -\frac{\omega^{4}}{64 \pi^{1 / 2}} \frac{l_{s}^{5}}{r^{2}} \\
& \times\left[-\left(30+20\left(\frac{2 r}{l_{s}}\right)^{2}+32\left(\frac{2 r}{l_{s}}\right)^{4}\right) e^{-4 r^{2} / l_{s}^{2}}+15 \sqrt{\pi}\left(\frac{2 r}{l_{s}}\right)^{-1} \operatorname{Erf}\left(\frac{2 r}{l_{s}}\right)\right]+\mathcal{O}\left(\omega^{6}\right), \\
\tilde{V}_{c, 2}(2 r)= & -\frac{\omega^{4} l_{s}}{32 \pi^{3 / 2}}\left[-\left(6+4\left(\frac{2 r}{l_{s}}\right)^{2}\right) e^{-4 r^{2} / l_{s}^{2}}+3 \sqrt{\pi}\left(\frac{2 r}{l_{s}}\right)^{-1} \operatorname{Erf}\left(\frac{2 r}{l_{s}}\right)\right]+\mathcal{O}\left(\omega^{6}\right), \\
\tilde{V}_{c, 4}(2 r)= & -\frac{\omega^{4}}{16 \pi^{5 / 2}} \frac{r^{2}}{l_{s}^{3}}\left[-2 e^{-4 r^{2} / l_{s}^{2}}+\sqrt{\pi}\left(\frac{2 r}{l_{s}}\right)^{-1} \operatorname{Erf}\left(\frac{2 r}{l_{s}}\right)\right]+\mathcal{O}\left(\omega^{6}\right), \\
\tilde{V}_{c, 6}(2 r)= & -\frac{\omega^{4}}{8 \pi^{7 / 2}} \frac{r^{4}}{l_{s}^{7}} \sqrt{\pi}\left(\frac{2 r}{l_{s}}\right)^{-1} \operatorname{Erf}\left(\frac{2 r}{l_{s}}\right)+\mathcal{O}\left(\omega^{6}\right) .
\end{aligned}
$$

For odd $p$, we obtain

$$
\begin{aligned}
& \tilde{V}_{c, 1}(2 r)=-\frac{\omega^{4}}{8 \pi} \frac{l_{s}^{4}}{r^{2}}\left[1-\left(1+\left(\frac{2 r}{l_{s}}\right)^{2}+\frac{1}{2}\left(\frac{2 r}{l_{s}}\right)^{4}\right) e^{-\left(2 r / l_{s}\right)^{2}}\right]+\mathcal{O}\left(\omega^{6}\right), \\
& \tilde{V}_{c, 3}(2 r)=-\frac{\omega^{4}}{16 \pi^{2}}\left[1-\left(1+\left(\frac{2 r}{l_{s}}\right)^{2}\right) e^{-\left(2 r / l_{s}\right)^{2}}\right]+\mathcal{O}\left(\omega^{6}\right), \\
& \tilde{V}_{c, 5}(2 r)=-\frac{\omega^{4}}{16 \pi^{3}} \frac{r^{2}}{l_{s}^{4}}\left(1-e^{-\left(2 r / l_{s}\right)^{2}}\right)+\mathcal{O}\left(\omega^{6}\right) .
\end{aligned}
$$

Note that the appearance of the factor $r^{-2}$ for $p=0,1$ does not imply the blow up of the effective potentials for small $r$. This is because our calculations so far are valid under the assumptions $r \omega, \omega l_{s}^{2} / r \ll 1$, and therefore, $r$ cannot be extremely small.

\section{Shape of the effective potential}

In the previous sections, we have explicitly evaluated the integrals to obtain the effective potentials $\tilde{V}_{p}(2 r)$ approximately for small $\omega$. In this section, we investigate the shape of the effective potential $U_{p}(r)$, which includes the effect of the centrifugal force. The formulas are valid as long as $\omega<r / l_{s}^{2}$ and $\omega r<1$ are satisfied. In the following, we focus our attention on the leading contribution for small $\omega$, that is, the contributions of order $\mathcal{O}\left(\omega^{2}\right)$. As long as $r$ is small compared to $l_{s}$, the SUGRA contribution $\tilde{V}_{c, p}(2 r)$ is negligible. Note that for larger $r$, the SYM contributions $\tilde{V}_{o, p}(2 r)$ decay exponentially, and the potential is dominated by the SUGRA contributions.

We are interested in a possibility of two $\mathrm{D} p$-banes to form a stable (resonant) state with a length scale dynamically determined by the revolving motion. Our strategy to investigate such a possibility is the following. Analogous to the classical mechanics with the Newtonian potential, we consider

$$
U_{p}(r):=\tilde{V}_{p}(2 r)+V_{\text {cent }, p}(r),
$$


where $V_{\text {cent }, p}(r)$ is the centrifugal potential for the $\mathrm{D} p$-branes. If there is a local minimum for $U_{p}(r)$, there could be a non-trivial state which is stable, at least when the quantum tunneling effect is ignored. Since the lowest energy state consisting of two $\mathrm{D} p$-branes is the static one which is half-BPS, the non-trivial state, if exists, should have positive energy.

In order to study this, we need to take into account the effect of the centrifugal force. The centrifugal potential $V_{\text {cent, } p}(r)$ is derived from the SYM action with the revolving background $B_{I}$ for the adjoint scalar fields $\Phi_{I}$. The background part of the SYM action in the Lorentzian signature is given by

$$
-\frac{1}{2 g_{\mathrm{YM}}^{2}} \int d^{p+1} x \operatorname{Tr}\left(\partial_{\mu} B_{I}\right)^{2}+\cdots
$$

where the revolving $\mathrm{D} p$-brane is described by

$$
B_{8}=r \cos (\omega t) \cdot \sigma_{3} / l_{s}^{2}, \quad B_{9}=r \sin (\omega t) \cdot \sigma_{3} / l_{s}^{2} .
$$

Substituting these backgrounds into the action, we obtain

$$
V_{\text {cent }, p}(r, \omega)=\frac{r^{2} \omega^{2}}{g_{\mathrm{YM}}^{2} l_{s}^{4}}=r^{2} \omega^{2} T_{p}
$$

This becomes a more familiar form when we fix the angular momentum per volume $L=$ $2 r^{2} \omega T_{p}$ and replace $\omega$ with $L / 2 r^{2} T_{p}$. To indicate the $g_{s}$-dependence explicitly, we denote $T_{p}=a_{p} / g_{s}$ where $a_{p}:=(2 \pi)^{(1-p) / 2} l_{s}^{-p-1}$. Accordingly, we define $L_{0}:=L g_{s}$ which is independent of $g_{s}$. Then, the relation for the angular momentum can be rewritten as $L_{0}=2 r^{2} \omega a_{p}$. Replacing $\omega$ with $L_{0} / 2 r^{2} a_{p}$ in the $V_{\text {cent }, p}(r)$, we obtain

$$
U_{p}(r)=\frac{L_{0}^{2}}{4 a_{p} g_{s} r^{2}}+\left.\tilde{V}_{p}(r)\right|_{\omega=L_{0} / 2 r^{2} a_{p}} .
$$

For large $r$, we know from the SUGRA analysis that $\tilde{V}_{p}(r)$ behaves as $-r^{p-7}$. This is much weaker than $V_{\text {cent }, p}(r)$ and $U_{p}(r)$ is repulsive. Therefore resonant states, if exist, should be formed for small $r$ region where SYM contributions are important.

The plots for various $p$ are given in figure 2. In the figure, we set $T_{p}=1$ and $L=0.01$. We find that the potential $U_{p}(r)$ is always repulsive for $p \geq 3$, even for small $r$. This implies that no resonant states are anticipated for $p \geq 3$. For $p=0,1$ and 2 , the situation is different and more interesting. For those cases, the potential changes its behavior and becomes attractive for small enough $r$. This may indicate the existence of a resonant state. Interestingly, such a state was discussed in $[7,8]$. The behavior of our potential seems to be consistent with their result.

\subsection{Multiple D $p$-branes}

We can generalize the previous results of $U_{p}(r)$ to $U_{N, p}(r)$, where a pair of stacks of $N \mathrm{D} p$ branes is considered instead of a pair of just a single $\mathrm{D} p$-brane. Then the number of open strings stretched between the pair becomes $N^{2}$ and thus $\tilde{V}_{p}(2 r)$ is multiplied by $N^{2}$, while 


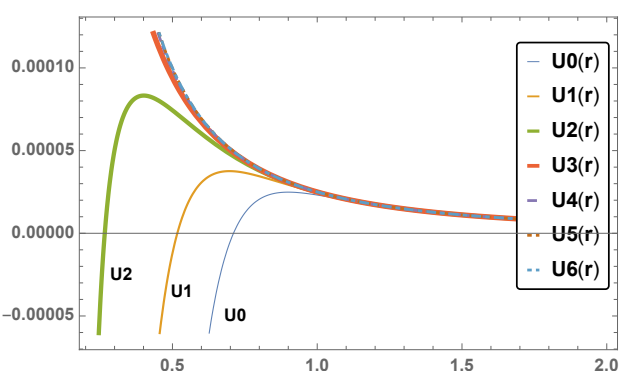

Figure 2. The potential $U_{p}(r)$ with $T_{p}=1$ and $L=0.01$.

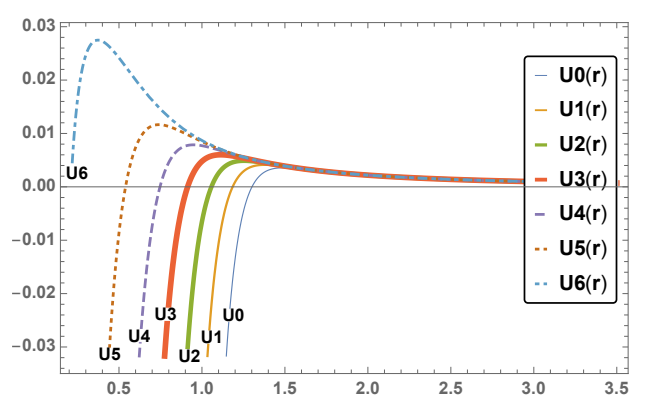

Figure 3. The potential $U_{N, p}(r)$ with $T_{p}=1, L=0.01$ and $N=350$.

the centrifugal potential $V_{\text {cent,p }}(r)$ is simply multiplied by $N$. As a result, the potential becomes

$$
U_{N, p}(r):=\frac{N L_{0}^{2}}{4 a_{p} g_{s} r^{2}}+N^{2} \tilde{V}_{p}(2 r)
$$

The shapes of $U_{N, p}(r)$ are depicted in figure 3. Here also we set $T_{p}=1, L=0.01$ and $N=350$. We can see that, for a sufficiently large $N$, the potential becomes negative and falls off for smaller $r$.

To see the behavior of $U_{N, p}(r)$ for small $r$ in more detail, we expand $U_{N, p}(r)$ with respect to $r$. Since $\tilde{V}_{p}(2 r)$ was determined by expanding in terms of $\omega$, the further $r$-expansion is valid as long as $\omega \ll r / l_{s}^{2}$ is satisfied.

Recall that the leading order of $\tilde{V}_{p}(2 r)$ is given by

$$
\tilde{V}_{p}(2 r)=-\frac{4}{\pi}(4 \pi)^{-\frac{p}{2}} \frac{\sqrt{\pi}}{\Gamma\left(\frac{p+1}{2}\right)} \omega^{2} l_{s}^{1-p}\left(\frac{2 r}{l_{s}}\right)^{2} f_{p, 1}\left(\frac{2 r}{l_{s}}\right)+\mathcal{O}\left(\omega^{4}\right),
$$

where the analytic continuation of $\omega$ have performed. At small $x$, the function $f_{p, 1}(x)$ behaves as

$$
f_{p, 1}(x) \sim \begin{cases}\frac{\pi}{2 x}-\sqrt{\pi}, & (p=0) \\ \frac{1}{2} \log \frac{e^{-\gamma}}{x}, & (p=1) \\ \frac{1}{2} \Gamma\left(\frac{p-1}{2}\right) . & (p \geq 2)\end{cases}
$$

For the details, see appendix C. Thus, for $p \geq 2$, we have $V_{p}(2 r) \propto \omega^{2} r^{2}$. For $p=0,1$, it has a different $r$-dependence; $V_{0}(2 r) \propto \omega^{2} r$ and $V_{1}(2 r) \propto \omega^{2} r^{2} \log r$.

Now we discuss competition between $\tilde{V}_{p}(2 r)$ and $V_{\text {cent }, p}(r)$. Let us first consider $p \geq 2$ cases. We have $N^{2} \tilde{V}_{p}(2 r) \sim-c_{p} N^{2} \omega^{2} r^{2}$ for small $r$, where

$$
c_{p}:=\frac{32}{p-1}(4 \pi)^{-(p+1) / 2} l_{s}^{-p-1} .
$$

For a fixed angular momentum, the potential $\tilde{V}_{p}(2 r)$ becomes

$$
N^{2} \tilde{V}_{p}(2 r) \sim-\frac{c_{p} N^{2}}{a_{p}} \frac{L_{0}^{2}}{4 a_{p} r^{2}} .
$$


Therefore, the potential $U_{N, p}(r)$ is

$$
U_{N, p}(r) \sim\left(\frac{1}{g_{s}}-\frac{c_{p} N}{a_{p}}\right) \frac{N L_{0}^{2}}{4 a_{p} r^{2}}
$$

If this is negative, then the attractive interaction wins for small $r$. We find that the 't Hooft coupling $g_{s} N$ governs the sign of the potential for small $r$. Indeed, if the following condition is satisfied,

$$
g_{s} N>\frac{a_{p}}{c_{p}}=2^{-(7-p) / 2}(p-1) \pi
$$

the potential becomes negative for small $r$. The right-hand side takes the smallest value $2^{-5 / 2} \pi=0.555$ for $p=2$. Therefore, we need an $\mathcal{O}(1)$ value for $g_{s} N$ if we would like to have an attractive force for small $r$, which is a necessary condition for a resonant state to be formed. The requirement $g_{s} N=\mathcal{O}(1)$ suggests that the backreaction of the D-brane system to the space-time metric may not be neglected. Indeed, since the Newtonian potential created by $N \mathrm{D} p$-branes is roughly given by the form (assuming that the dimension is adjusted by $l_{s}$ )

$$
\frac{G_{\mathrm{N}} T_{p}}{r^{7-p}} \sim \frac{g_{s} N}{r^{7-p}}
$$

a typical scale of the curvature of the space-time is given by $\left(g_{s} N\right)^{1 /(7-p)} l_{s}$. Thus in order to discuss the existence of resonant states in a controlled manner, it will be necessary to consider curved background space-time in which D-branes are revolving.

Next, let us consider a case of $p=0$. The potential becomes

$$
U_{0} \sim\left(\frac{1}{g_{s}}+\frac{16 N}{\sqrt{2} \pi}-\frac{4 N}{\sqrt{2 \pi} r / l_{s}}\right) \frac{N L_{0}^{2}}{4 a_{0} r^{2}} .
$$

Remarkably, the potential becomes attractive around $r / l_{s} \sim g_{s} N$ for any value of $g_{s} N$. Therefore, by taking $g_{s} N$ to be small, our analysis can be justified.

Finally, the potential for $p=1$ turns out to be

$$
U_{1} \sim\left(\frac{1}{g_{s}}-\frac{4 N}{\pi} \log \frac{e^{-\gamma} l_{s}}{2 r}\right) \frac{N L_{0}^{2}}{4 a_{1} r^{2}} .
$$

This becomes attractive for a region of $r$ smaller than

$$
r_{c} \sim \frac{1}{2} \exp \left(-\frac{\pi}{4 g_{s} N}-\gamma\right) l_{s} .
$$

It is curious that the right-hand side has a non-perturbative form for $g_{s} N$.

It is interesting that the potential becomes always negative for $p=0$ and $p=1$ for small $r$, which indicates the existence of resonant states of two revolving $\mathrm{D} p$-branes.

\section{Conclusions and discussions}

In this paper, we have obtained the effective potential of revolving $\mathrm{D} p$-branes. The interactions between D-branes can be evaluated either by closed string exchange for long distance 
$r>l_{s}$ or by open string 1-loop amplitude for short distance $r<l_{s}$, and they are related by a modular transformation. In order to evaluate the effective potential interpolating these two regions, we used the technique of partial modular transformation to sum up both of open and closed string contributions without a double counting. The shape of the effective potential $U_{N, p}(r)$, which includes the effect of the centrifugal force, is drawn for small angular frequency $\omega$ in a nonrelativistic region. The interaction is repulsive for large $r$, but we find that it can become attractive for small $r$.

The shapes are different between $p=0,1$ and $p \geq 2$. Since the infrared effects are stronger for $p=0,1$, the potential falls down faster around $r \sim 0$. Especially for $p=1$, there is an additional logarithmic factor $\log (r)$ in the effective potential and the typical distance $r=r_{c}$ where the interaction changes from repulsive $\left(r>r_{c}\right)$ to attractive $\left(r<r_{c}\right)$ is given in a nonperturbative form $r_{c} \propto \exp \left(-\pi / 4 g_{s} N\right) l_{s}$. On the other hand, for $p \geq 2$, the typical shape of the potential is given by $-c / r^{2}$ for small $r$. Thus it is classically unstable. Quantum mechanically, depending on the numerical coefficient $c>0$, the system is either unstable or has a bound state [17]. In our system the potential is more complicated, since it becomes positive in an intermediate region of $r$, and we need more detailed analysis to see whether it has a metastable bound state or not. Furthermore, in the paper, we have evaluated the integral in $U_{N, p}(r)$ for small $r$ by using $\omega / r$ expansions. Thus angular frequency must be small, $\omega<r / l_{s}^{2}$, and the approximation is not valid for $r<l_{s}^{2} \omega$.

In the following discussion, we look at the behavior of the potential at $r \sim 0$ by using another expansion with respect to $r / \omega$. First we can easily see that the effective potential vanishes at $r=0 ; \tilde{V}_{o}(0)=\tilde{V}_{B, p}(0)+\tilde{V}_{F, p}(0)=0$ by shifting the integration variable, $k_{\tau} \rightarrow k_{\tau} \pm \omega$. Thus, the unstable behavior in the $\omega / r$ expansion may be merely ostensible. In order to further understand the behavior at $r \sim 0$, let us go back to the calculation of the bosonic part, eq. (2.5). It consists of integrals

$$
-\int_{\Lambda^{-2}}^{\infty} \frac{d t}{t} \int \frac{d^{p+1} k}{(2 \pi)^{p+1}} e^{-t f(k, \theta)}
$$

with a singular function at $(k, r) \sim(0,0)$ :

$$
f(k, \theta):=k^{2}+4 r^{2}+\omega^{2}-\frac{8(r \omega)^{2}}{k^{2}+4 r^{2}}-\sqrt{4 \omega^{2} \cos ^{2} \theta k^{2}+\left(\frac{8(r \omega)^{2}}{k^{2}+4 r^{2}}\right)^{2}},
$$

where we set $k_{\tau}=k \cos \theta$. Around $k=0$, this behaves as

$$
f(k)=4 r^{2}-3 \omega^{2}+\left(1-\cos ^{2} \theta+\frac{\omega^{2}}{r^{2}}\right) k^{2}+\mathcal{O}\left(k^{4}\right) .
$$

Note that $f(k)$ becomes negative when $\omega / r>2 / \sqrt{3}$, which implies that the $t$-integral diverges. To illustrate this, suppose that $\omega / r$ is large. Then, the above integral can be estimated for large $t$ as

$$
\begin{aligned}
& -\int^{\infty} \frac{d t}{t} \int \frac{d^{p+1} k}{(2 \pi)^{p+1}} e^{-t\left(f(0)+\left(1-\cos ^{2} \theta+(\omega / r)^{2}\right) k^{2}\right)} \\
& \sim-(4 \pi)^{(p+1) / 2}\left(\frac{r}{\omega}\right)^{p+1} \int^{\infty} \frac{d t}{t} t^{-(p+1) / 2} e^{-t f(0)}
\end{aligned}
$$


Indeed, this integral diverges when $f(0)=4 r^{2}-3 \omega^{2}<0$. Recall that, if we fix the angular momentum $L$, then $\omega$ grows as $r^{-2}$. Therefore, the potential $U_{N, p}(r)$ has a problem for too small $r$ with fixed $L$.

To see what happens at $\omega / r=2 / \sqrt{3}$, consider the function

$$
\int_{1}^{\infty} \frac{d t}{t} t^{-(p+1) / 2} e^{-z t}=E_{(p+3) / 2}(z)
$$

of a complex variable $z$. The integral in the left-hand side is well-defined for $\operatorname{Re}(z)>0$. We find that $z=0$ is a branch point. The non-analytic part turns out to be

$$
E_{(p+3) / 2}(z) \sim \begin{cases}-\frac{(-z)^{n+1}}{(n+1) !} \log z, & (p=2 n+1) \\ (-1)^{n+1} \frac{\pi}{\Gamma\left(n+\frac{3}{2}\right)} z^{n+\frac{1}{2}} . & (p=2 n)\end{cases}
$$

Therefore, if we define $U_{N, p}(r)$ for small $r$ via the analytic continuation, then it takes complex value for $\omega / r>2 / \sqrt{3}$. This may be a signal of an instability of the system. Since this behavior comes from the fact that the integral (5.1) is not well-defined for small momentum $k$, it is tempting to speculate that the shape of the D-branes might be deformed due to the revolving motion. Finally we note that this kind of instability does not occur for the fermionic part of the effective action $\tilde{V}_{F, p}(2 r)$. It is specific to the bosonic part in which the kinetic terms of the gauge bosons and scalars are mixed due to the revolving motion. This situation is similar to [18]. The effective potential vanishes just at $r=0$, but the instability itself seems to be present. We want to come back to the origin of the instability in future investigations.

\section{Acknowledgments}

This work is supported in part by Grants-in-Aid for Scientific Research No. 16K05329, No. 18H03708 and No. 19K03851 from the Japan Society for the Promotion of Science.

\section{A Various functions for SYM contributions}

In subsection 3.1, we need to determine the explicit form of functions defined as

$$
f_{p, m}(x):=x^{1+p-2 m} \int_{0}^{\infty} d k e^{-x^{2}\left(k^{2}+1\right)} \frac{k^{p}}{\left(k^{2}+1\right)^{m}} .
$$

We can rewrite them as

$$
\begin{aligned}
f_{p, m}(x) & =x^{1+p-2 m} \int_{0}^{\infty} d k e^{-x^{2}\left(k^{2}+1\right)} \frac{k^{p-2}\left(k^{2}+1\right)-k^{p-2}}{\left(k^{2}+1\right)^{m}} \\
& =f_{p-2, m-1}(x)-x^{2} f_{p-2, m}(x) .
\end{aligned}
$$

These recurrence relations allow us to determine $f_{p, m}(x)$ from $f_{0, m}(x)$ or $f_{1, m}(x)$ according to whether $p$ is even or odd, respectively. Note that, to obtain $f_{p, m}(x)$ for $p \geq 2$ and $-1 \leq m \leq 4$, we need $f_{0, m}(x)$ or $f_{1, m}(x)$ with $m<-1$. 


\section{A.1 $f_{0, m}(x)$}

For $m=0$, this is just a Gaussian integral,

$$
f_{0,0}(x)=\frac{\sqrt{\pi}}{2} e^{-x^{2}} .
$$

The functions $f_{0, m}(x)$ satisfy

$$
f_{0, m-1}(x)=-\frac{1}{2} x^{2-2 m} \frac{d}{d x}\left(x^{2 m-1} f_{m}(x)\right) .
$$

From this relation, we obtain $f_{0, m}(x)$ for $m<0$ easily from $f_{0,0}(x)$. For example,

$$
\begin{aligned}
& f_{0,-1}(x)=\frac{\sqrt{\pi}}{4}\left(1+2 x^{2}\right) e^{-x^{2}}, \\
& f_{0,-2}(x)=\frac{\sqrt{\pi}}{8}\left(3+4 x^{2}+4 x^{4}\right) e^{-x^{2}}, \\
& f_{0,-3}(x)=\frac{\sqrt{\pi}}{16}\left(15+18 x^{2}+12 x^{4}+8 x^{6}\right) e^{-x^{2}}, \\
& f_{0,-4}(x)=\frac{\sqrt{\pi}}{32}\left(105+120 x^{2}+72 x^{4}+32 x^{6}+16 x^{8}\right) e^{-x^{2}} .
\end{aligned}
$$

Next, we consider $m>0$. By integrating (A.4), we obtain

$$
f_{0,1}(x)=\frac{\pi}{2 x}(1-\operatorname{Erf}(x))
$$

where

$$
\operatorname{Erf}(x):=\frac{2}{\sqrt{\pi}} \int_{0}^{x} d t e^{-t^{2}},
$$

and the integration constant is specified by $\lim _{x \rightarrow \infty} f_{m}(x)=0$ which is obvious from the original integral form (A.1). The functions with $m \geq 2$ can be obtained similarly. It is also convenient to use the following recurrence relations

$$
f_{0, m}(x)=\frac{1}{x^{2}}\left(1-\frac{1+2 x^{2}}{2(m-1)}\right) f_{0, m-1}(x)+\frac{1}{(m-1) x^{2}} f_{0, m-2}(x) .
$$

This can be derived as follows;

$$
\begin{aligned}
f_{0, m}(x) & =x^{1-2 m} \int_{0}^{\infty} d k e^{-x^{2}\left(k^{2}+1\right)} \frac{\left(k^{2}+1\right)-k^{2}}{\left(k^{2}+1\right)^{m}} \\
& =\frac{1}{x^{2}} f_{0, m-1}(x)-x^{1-2 m} \int_{0}^{\infty} d k e^{-x^{2}\left(k^{2}+1\right)} \frac{k^{2}}{\left(k^{2}+1\right)^{m}},
\end{aligned}
$$

where the last integral can be calculated as

$$
\begin{aligned}
- & x^{1-2 m} \int_{0}^{\infty} d k e^{-x^{2}\left(k^{2}+1\right)} \frac{k^{2}}{\left(k^{2}+1\right)^{m}} \\
& =-\frac{x^{1-2 m}}{2(m-1)} \int_{0}^{\infty} d k e^{-x^{2}\left(k^{2}+1\right)} \frac{1-2 x^{2} k^{2}}{\left(k^{2}+1\right)^{m-1}} \\
& =-\frac{1}{2(m-1) x^{2}} f_{0, m-1}(x)+\frac{1}{(m-1) x^{2}} f_{0, m-2}(x)-\frac{1}{m-1} f_{0, m-1}(x) .
\end{aligned}
$$


We can obtain

$$
\begin{aligned}
& f_{0,2}(x)=\frac{\sqrt{\pi}}{2 x^{2}} e^{-x^{2}}+\frac{\pi}{4 x^{3}}\left(1-2 x^{2}\right)(1-\operatorname{Erf}(x)) \\
& f_{0,3}(x)=\frac{\sqrt{\pi}}{8 x^{4}} e^{-x^{2}}\left(3-2 x^{2}\right)+\frac{\pi}{16 x^{5}}\left(3-4 x^{2}+4 x^{4}\right)(1-\operatorname{Erf}(x)) \\
& f_{0,4}(x)=\frac{\sqrt{\pi}}{48 x^{6}} e^{-x^{2}}\left(15-8 x^{2}+4 x^{4}\right)+\frac{\pi}{96 x^{7}}\left(15-18 x^{2}+12 x^{4}-8 x^{6}\right)(1-\operatorname{Erf}(x)) .
\end{aligned}
$$

\section{A.2 $f_{1, m}(x)$}

This can be written as

$$
f_{1, m}(x)=x^{2-2 m} \int_{0}^{\infty} d k e^{-x^{2}\left(k^{2}+1\right)} \frac{k}{\left(k^{2}+1\right)^{m}}=\frac{1}{2} x^{2-2 m} E_{m}\left(x^{2}\right),
$$

where

$$
E_{m}(x):=\int_{1}^{\infty} d t t^{-m} e^{-x t}
$$

For $m \geq 1, E_{m+1}(x)$ satisfy

$$
E_{m+1}(x)=-\frac{x}{m} E_{m}(x)+\frac{1}{m} e^{-x} .
$$

This recurrence relation can be solved in terms of $E_{1}(x)$. The solution is

$$
E_{m+1}(x)=\frac{(-x)^{m}}{m !} E_{1}(x)-\frac{e^{-x}}{x} \sum_{l=1}^{m} \frac{(m-l) !}{m !}(-x)^{l} .
$$

By using them, we obtain

$$
\begin{aligned}
f_{1,1}(x) & =\frac{1}{2} E_{1}\left(x^{2}\right) \\
f_{1,2}(x) & =\frac{1}{2 x^{2}} e^{-x^{2}}-\frac{1}{2} E_{1}\left(x^{2}\right), \\
f_{1,3}(x) & =\frac{1-x^{2}}{4 x^{4}} e^{-x^{2}}+\frac{1}{4} E_{1}\left(x^{2}\right), \\
f_{1,4}(x) & =\frac{2-x^{2}+x^{4}}{12 x^{6}}-\frac{1}{12} E_{1}\left(x^{2}\right) .
\end{aligned}
$$

For $m \leq 0$, we use

$$
E_{0}(x)=\frac{1}{x} e^{-x}, \quad E_{m-1}(x)=-\frac{d}{d x} E_{m}(x) .
$$

From them, we obtain

$$
\begin{aligned}
f_{1,0}(x) & =\frac{1}{2} e^{-x^{2}} \\
f_{1,-1}(x) & =\frac{1}{2}\left(1+x^{2}\right) e^{-x^{2}} \\
f_{1,-2}(x) & =\frac{1}{2}\left(2+2 x^{2}+x^{4}\right) e^{-x^{2}} \\
f_{1,-3}(x) & =\frac{1}{2}\left(6+6 x^{2}+3 x^{4}+x^{6}\right) e^{-x^{2}} \\
f_{1,-4}(x) & =\frac{1}{2}\left(24+24 x^{2}+12 x^{4}+4 x^{6}+x^{8}\right) e^{-x^{2}} .
\end{aligned}
$$




\section{B Various functions for SUGRA contributions}

For the calculations of the SUGRA contributions $\tilde{V}_{c}(2 r)$, we need to determine

$$
g_{\alpha}(x):=\int_{1}^{\infty} d s s^{-\alpha} e^{-x^{2} / s},
$$

where $\alpha:=(9-p) / 2$. The recurrence relations

$$
\begin{aligned}
g_{\alpha}(x) & =\int_{0}^{1} d t t^{\alpha-2} e^{-x^{2} t} \\
& =-\left.\frac{1}{x^{2}} t^{\alpha-1} e^{-x^{2} t}\right|_{0} ^{1}+\frac{\alpha-2}{x^{2}} \int_{0}^{1} d t t^{\alpha-3} e^{-x^{2} t} \\
& =-\frac{1}{x^{2}} e^{-x^{2}}+\frac{\alpha-2}{x^{2}} g_{\alpha-1}(x)
\end{aligned}
$$

for $\alpha>2$ imply that we only need to determine $g_{3 / 2}(x)$ or $g_{2}(x)$, depending on whether $p$ is even or odd, respectively.

\section{B.1 $g_{3 / 2}(x)$}

This can be rewritten as

$$
g_{3 / 2}(x)=\int_{1}^{\infty} d s s^{-3 / 2} e^{-x^{2} / s}=\frac{\sqrt{\pi}}{x} \operatorname{Erf}(x) .
$$

Then, the solutions of the recurrence relations (B.2) is

$$
g_{m+3 / 2}(x)=\frac{\Gamma(m+1 / 2)}{x^{2 m+1}} \operatorname{Erf}(x)-\frac{e^{-x^{2}}}{x^{2}} \sum_{l=0}^{m-1} \frac{\Gamma(m+1 / 2)}{\Gamma(m-l+1 / 2)} x^{-2 l} .
$$

Explicitly,

$$
\begin{aligned}
& g_{5 / 2}(x)=\frac{\sqrt{\pi}}{2 x^{3}} \operatorname{Erf}(x)-\frac{1}{x^{2}} e^{-x^{2}}, \\
& g_{7 / 2}(x)=\frac{3 \sqrt{\pi}}{4 x^{5}} \operatorname{Erf}(x)-\frac{3+2 x^{2}}{2 x^{4}} e^{-x^{2}}, \\
& g_{9 / 2}(x)=\frac{15 \sqrt{\pi}}{8 x^{7}} \operatorname{Erf}(x)+\frac{15+10 x^{2}+4 x^{4}}{4 x^{6}} e^{-x^{2}} .
\end{aligned}
$$

\section{B.2 $g_{2}(x)$}

This is simply

$$
g_{2}(x)=\frac{1-e^{-x^{2}}}{x^{2}}
$$

Thus, for $m \geq 1, g_{m+2}(x)$ is given as

$$
g_{m+2}(x)=\frac{m !}{x^{2 m+2}}-\frac{e^{-x^{2}}}{x^{2}} \sum_{l=0}^{m} \frac{m !}{(m-l) !} x^{-2 l} .
$$

Explicitly,

$$
\begin{aligned}
& g_{3}(x)=\frac{1}{x^{4}}-\frac{1+x^{2}}{x^{4}} e^{-x^{2}} \\
& g_{4}(x)=\frac{2}{x^{6}}-\frac{2+2 x^{2}+x^{4}}{x^{6}} e^{-x^{2}}
\end{aligned}
$$




\section{C $\mathcal{O}\left(\omega^{2}\right)$ contributions for small $r$}

The effective potential $\tilde{V}_{p}(2 r)$ at the leading order in $\omega$ is given by $f_{p, 1}(x)$. They can be determined recursively, as explained in appendix A. When we are only interested in the leading order terms in $f_{p, 1}(x)$ with respect to $x$, they can be derived more easily as follows.

It is easy to obtain

$$
f_{p, 0}(x)=\frac{1}{2} \Gamma\left(\frac{p+1}{2}\right) e^{-x^{2}} .
$$

Then, the recurrence relation (A.2) becomes

$$
f_{p, 1}(x)=\frac{1}{2} \Gamma\left(\frac{p-1}{2}\right) e^{-x^{2}}-x^{2} f_{p-2,1}(x) .
$$

First, we assume that $p$ is even. We found in appendix A that

$$
\begin{aligned}
& f_{0,1}(x)=\frac{\pi}{2 x}(1-\operatorname{Erf}(x))=\frac{\pi}{2 x}-\sqrt{\pi}+\mathcal{O}\left(x^{2}\right), \\
& f_{2,1}(x)=\frac{\sqrt{\pi}}{2} e^{-x^{2}}-x^{2} f_{0,1}(x)=\frac{\sqrt{\pi}}{2}+\mathcal{O}(x) .
\end{aligned}
$$

Recursively, we can show that $f_{0,1}(x)=\mathcal{O}(1)$ for $p \geq 2$. Note that the qualitative behavior is different only for $p=0$.

For odd $p$,

$$
\begin{aligned}
& f_{1,1}(x)=\frac{1}{2} E_{1}\left(x^{2}\right)=-\frac{1}{2}(\gamma+\log x)+\mathcal{O}(x), \\
& f_{3,1}(x)=\frac{1}{2} e^{-x^{2}}-x^{2} f_{1,1}(x)=\frac{1}{2}+\mathcal{O}\left(x^{2} \log x\right) .
\end{aligned}
$$

Recursively, we can show that $f_{p, 1}(x)=\mathcal{O}(1)$ for $p \geq 3$. We find that there exists a logarithmic correction to the leading behavior for $p=1$. In summary, the leading behavior of $f_{p, 1}(x)$ is

$$
f_{p, 1}(x) \sim \begin{cases}\frac{\pi}{2 x}-\sqrt{\pi}, & (p=0) \\ \frac{1}{2} \log \frac{e^{-\gamma}}{x}, & (p=1) \\ \frac{1}{2} \Gamma\left(\frac{p-1}{2}\right) . & (p \geq 2)\end{cases}
$$

Open Access. This article is distributed under the terms of the Creative Commons Attribution License (CC-BY 4.0), which permits any use, distribution and reproduction in any medium, provided the original author(s) and source are credited.

\section{References}

[1] S. Iso, N. Kitazawa, H. Ohta and T. Suyama, Dynamics of Revolving D-branes at Short Distances, JHEP 01 (2020) 182 [arXiv:1909.10717] [INSPIRE].

[2] R. Blumenhagen, B. Körs, D. Lüst and S. Stieberger, Four-dimensional String Compactifications with D-branes, Orientifolds and Fluxes, Phys. Rept. 445 (2007) 1 [hep-th/0610327] [INSPIRE]. 
[3] L.E. Ibanez and A.M. Uranga, String theory and particle physics: An introduction to string phenomenology, Cambridge University Press, Cambridge U.K. (2012) [INSPIRE].

[4] D. Baumann and L. McAllister, Inflation and String Theory, Cambridge Monographs on Mathematical Physics, Cambridge University Press (2015) [DOI] [arXiv:1404.2601] [INSPIRE].

[5] S. Iso and N. Kitazawa, Revolving D-branes and Spontaneous Gauge Symmetry Breaking, PTEP 2015 (2015) 123B01 [arXiv:1507.04834] [INSPIRE].

[6] S. Iso and N. Kitazawa, A Possibility of Lorentz Violation in the Higgs Sector, Mod. Phys. Lett. A 35 (2019) 2050064 [arXiv: 1812.08912] [INSPIRE].

[7] U.H. Danielsson, G. Ferretti and B. Sundborg, D particle dynamics and bound states, Int. J. Mod. Phys. A 11 (1996) 5463 [hep-th/9603081] [INSPIRE].

[8] D.N. Kabat and P. Pouliot, A Comment on zero-brane quantum mechanics, Phys. Rev. Lett. 77 (1996) 1004 [hep-th/9603127] [INSPIRE].

[9] J. Polchinski, String theory. Vol. 1: An introduction to the bosonic string, Cambridge Monographs On Mathematical Physics, Cambridge University Press, Cambridge, U.K. (1998) [DOI] [INSPIRE].

[10] J. Polchinski, String theory. Vol. 2: Superstring theory and beyond, Cambridge Monographs On Mathematical Physics, Cambridge University Press, Cambridge, U.K. (1998) [DOI] [INSPIRE].

[11] C. Bachas, D-brane dynamics, Phys. Lett. B 374 (1996) 37 [hep-th/9511043] [InSPIRE].

[12] G. Lifschytz, Comparing D-branes to black-branes, Phys. Lett. B 388 (1996) 720 [hep-th/9604156] [INSPIRE].

[13] S. Hirano and Y. Kazama, Scattering of closed string states from a quantized D particle, Nucl. Phys. B 499 (1997) 495 [hep-th/9612064] [INSPIRE].

[14] Y. Kazama, Scattering of quantized Dirichlet particles, Nucl. Phys. B 504 (1997) 285 [hep-th/9705111] [INSPIRE].

[15] S. Iso, H. Ohta and T. Suyama, Effective Potential for Revolving D-branes, JHEP 04 (2019) 151 [arXiv: 1812.11505] [INSPIRE].

[16] M.R. Douglas, D.N. Kabat, P. Pouliot and S.H. Shenker, D-branes and short distances in string theory, Nucl. Phys. B 485 (1997) 85 [hep-th/9608024] [INSPIRE].

[17] L.D. Landau and E.M. Lifshits, Quantum Mechanics: Non-Relativistic Theory, Course of Theoretical Physics, vol. 3, Butterworth-Heinemann, Oxford (1991) [INSPIRE].

[18] K. Hashimoto and S. Nagaoka, Recombination of intersecting D-branes by local tachyon condensation, JHEP 06 (2003) 034 [hep-th/0303204] [INSPIRE]. 